\title{
Alcohólicos ingresados en una sala de psiquiatría: mortalidad a los 14 años
}

\section{Alcoholic patients hospitalized in a psychiatry unit: mortality at 14 years}

| Miquel Monrás; Lluisa Ortega
| Unitat d'Alcohologia (UA). Hospital Clínic de Barcelona (HCB)

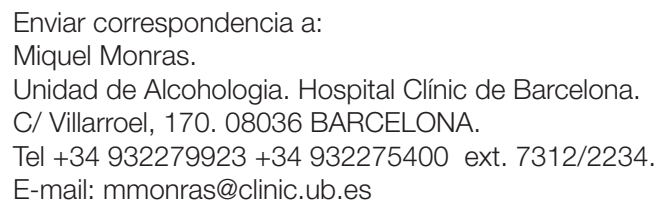

\section{RESUMEN}

Introducción: Los pacientes alcohólicos presentan una elevada mortalidad. Se conoce la de los pacientes que siguen un tratamiento ambulatorio especializado y la de los que ingresan por patologías orgánicas relacionadas con su abuso de alcohol, pero desconocemos si los alcohólicos con un perfil más psiquiátrico también fallecen de forma prematura. Objetivo: investigar las caracteristicas clínicas, evolución terapéutica, supervivencia y los factores que pueden predecir mejor su mortalidad posterior. Material: Seguimiento longitudinal a los 14 años de una cohorte de 91 pacientes alcohólicos ingresados en 1993 para desintoxicación en una Sala de Psiquiatría y que siguieron tratamiento ambulatorio posterior. Resultados: Presentan una elevada presencia de patología psiquiátrica $(40,6 \%)$ y múltiples antecedentes de tratamiento (78\%). A los 14 años la mortalidad ha sido del $34,1 \%$. Los pacientes fallecidos presentaban con más frecuencia deterioro cognitivo y situación de pensionistas. También recibian antidepresivos más a menudo, tenian menor soporte familiar y habian recaido más en el consumo de alcohol. Discusión: Muchos factores que predicen mayor mortalidad tienen relación con una mayor edad. En los pacientes más jóvenes la presencia de sintomas de deterioro neuropsicológico podría señalar una alteración orgánica prematura y probablemente un mayor riesgo de incumplimiento de tratamientos, mal cuidado físico y situaciones traumáticas, fisicas y mentales, todo lo cual favorecería también la mortalidad prematura.

Palabras clave: alcoholismo, mortalidad, hospitalización, patología dual.

\section{ABSTRACT}

Introduction: Alcoholic patients show a high mortality rate. We know about the increased mortality of outpatients following specialized treatment for alcohol abuse and inpatients with organic diseases related to alcohol, but it is not clear whether alcoholics with a comorbid psychiatric profile also die prematurely. Objective: To investigate clinical characteristics, therapeutic evolution, survival, and factors that can better predict mortality. Materials: 14-year longitudinal monitoring of 91 patients hospitalized in 1993 for detoxification in a psychiatric unit and who subsequently received outpatient treatment. Results: Patients show a high prevalence of psychiatric disorders (40.6\%) and multiple periods of previous treatment (78\%). After 14 years the mortality rate was $34.1 \%$. Deceased patients more often presented cognitive decline and were more often on pensions. They were also taking more antidepressants, had less family support and were more likely to have relapsed into alcohol use. Discussion: Many of the factors that predict higher mortality are age-related. In younger patients, the presence of neuropsychological deterioration symptoms may indicate a premature organic disorder and probably greater risk of treatment failure, poor physical care and traumatic physical and mental situations, all of which would also increase the likelihood of premature mortality.

Key words: alcoholism, mortality, hospitalization, dual diagnosis. 


\section{INTRODUCCIÓN}

$\mathrm{E}$ alcohol es la causa de alrededor del 5\% de las muertes ocurridas en Catalunya ${ }^{1}$, siendo los varones los que más contribuyen a esta mortalidad debido a su mayor consumo de alcohol. Las causas traumáticas, como los accidentes de tráfico, son las que provocan más muertes entre las personas jóvenes y más años de vida potenciales perdidos 2. Parecida o peor situación se produce en el resto del mundo desarrollado ${ }^{3-6}$, en que las personas que beben abusivamente llegan a triplicar el riesgo de muerte.

Obviamente los pacientes alcohólicos concentran unos porcentajes de fallecimientos prematuros muy altos. Esta mortalidad está directamente relacionada con la persistencia de los consumos alcohólicos ${ }^{7}$ y con la gravedad y duración de la dependencia ${ }^{8}$.

Los pacientes alcohólicos que acuden voluntariamente a realizar un tratamiento ambulatorio especializado tienen una edad relativamente joven (alrededor de los 40 años), un entorno socio-familiar conservado y una adecuada motivación ${ }^{7}$. Pueden considerarse que son los pacientes alcohólicos de mejor pronóstico, pues cuentan con una mayor capacidad de modificar su conducta. En estos pacientes algunos estudios de seguimiento a largo plazo muestran una mortalidad del $15 \%$ a los 10 años $^{7}$ y del $32 \%$ a los 20 años de iniciar el tratamiento ${ }^{9}$.

En cambio en los enfermos alcohólicos más graves desde el punto de vista de sus consecuencias, la mortalidad se incrementa, debido a las complicaciones orgánicas causadas por el mantenimiento del consumo de alcohol durante años. Cuando en los hospitales generales ingresan enfermos por patologías orgánicas relacionadas con el consumo de alcohol, y se les detecta su alcoholismo por parte de los servicios sanitarios, lo hacen a edades superiores -entorno a los 50 años- ${ }^{10}$ y en fases muy avanzadas de la dependencia. En estos casos los tratamientos alcohológicos tienen escasa efectividad ${ }^{11,12}$. La mortalidad de estos pacientes Ilega al $30 \%$ a los 4 años de su detección ${ }^{12}$, falleciendo de promedio a una edad de tan sólo 57,5 años.

Sin embargo tenemos poca información acerca de la evolución de las personas alcohólicas con una situación intermedia. Entre los alcohólicos con muchos años de consumo de alcohol y patología orgánica que requiere hospitalizaciones y los alcohólicos más preservados, más jóvenes, sin alteraciones físicas, con buena motivación y que realizan tratamientos ambulatorios, se sitúan los pacientes alcohólicos que ingresan para desintoxicación en salas de psiquiatría, en los que su patologia orgánica no es muy avanzada, pero tienen altos porcentajes de trastornos psiquiátricos o neuropsicológicos, tratamientos fracasados y deterioro de su entorno socio-familiar ${ }^{13}$.

Estos pacientes alcohólicos con comorbilidad psiquiátrica están recibiendo una atención creciente en los últimos años, al haberse concienciado los clínicos de la importancia de tener en cuenta la detección y adecuado tratamiento de la patología dual como factor de riesgo para el éxito del tratamiento alcohológico. Por ello reciben tratamientos más espe- cíficos y a la vez con un abordaje más amplio de las diversas dificultades que genera su conducta.

Si los reingresos, la utilización de servicios de Urgencias, los accidentes y las patologías orgánicas guardan relación directa con la inestabilidad de la abstinencia ${ }^{7}$, es de suponer que la mayor dificultad que tienen los alcohólicos más graves desde el punto de vista psicopatológico en mantenerse abstinentes les provocará una mayor morbi-mortalidad.

Aunque existen evidencias de que diversos trastornos mentales, sobretodo el abuso de alcohol y la esquizofrenia, generan un riesgo de mortalidad aumentado ${ }^{14}$, no sabemos si este riesgo es menor cuando la enfermedad alcohólica es tratada específicamente.

Tampoco sabemos como evoluciona la mortalidad entre pacientes alcohólicos que además tienen comorbididad psiquiátrica, o si el seguimiento correcto de los tratamientos especializados contribuye a mejorar la esperanza de vida más que la mera indicación del tratamiento.

Y finalmente no sabemos si este exceso de mortalidad se debe a las enfermedades orgánicas provocadas por el consumo abusivo de alcohol, tal como la cirrosis hepática o las neoplasias buco-faríngeas, o bien la presencia de patología psiquiátrica puede, por si misma, contribuir a este incremento de la mortalidad.

Por todo lo anterior el objetivo principal del presente trabajo es conocer la mortalidad a los 14 años de una cohorte de pacientes alcohólicos con una elevada gravedad de su dependencia, que han ingresado para desintoxicación en una Sala de Psiquiatría de un hospital general, así como las posibles características diferenciales con otros grupos de alcohólicos tratados.

También se quiere estudiar si la psicopatología, los antecedentes de tratamientos fracasados y el deterioro neuropsicológico son posibles factores de mal pronóstico para esta mortalidad y conocer si el éxito del tratamiento alcohológico la mejora.

\section{MATERIAL Y MÉTODO}

Se realiza un estudio de seguimiento longitudinal prospectivo de una cohorte de 91 pacientes con dependencia alcohólica según criterios DSM-III-R a los 14 años después de su ingreso para desintoxicación hospitalaria en la Unidad de Alcohologia del Hospital Clínico de Barcelona (UA-HCB). Esta cohorte ya fue estudiada en parte a los 3 años de su alta hospitalaria ${ }^{13}$.

No se excluyen a los pacientes que presentaban también abuso o dependencia de otras drogas, que no completaron el ingreso hospitalario por fuga o alta voluntaria o que no siguieron tratamiento ambulatorio de seguimiento en la propia UA-HCB.

El ingreso se realizó en una Sala cerrada de Psiquiatría de un hospital general. Su duración media fue de 21 dias, durante los cuales, además de la desintoxicación se inició un proceso de deshabituación, concienciación y entrenamiento 
en estrategias de afrontamiento mediante una progresiva salida de cada paciente a su medio social.

Entre otros aspectos clínicos se valoró, siguiendo criterios DSM-III-R, la presencia de otros trastornos psicopatológicos independientes del alcoholismo (especialmente del Eje II).

El deterioro cognitivo que presentan estos pacientes no es muy avanzado y habitualmente no llega a cumplir criterios DSM-III-R de demencia, o de trastorno amnésico persistente inducido por substancias. Por ello para su evaluación se utilizaron vías diversas en función de la situación de cada paciente. En algunos casos se usaron técnicas de neuroimagen (TAC craneales) o baterías neuropsicológicas -Escala de Inteligencia para Adultos de Wechsler (WAIS), Escala de Memoria de Wechsler (WMS), Halstead-Reitan-. En todos los pacientes existían síntomas indicativos: déficit de memoria, fabulaciones, cambios inapropiados de humor, incongruencias y contradicciones en la elaboración de la anamnesis, dificultades de comprensión y cumplimiento de las indicaciones terapéuticas, dificultades para una conducta diaria integrada en su medio o cambios en el comportamiento. Con frecuencia también existía antecedentes de delírium por abstinencia, encefalopatía hepática y neuropatía

Diez y nueve $(20,8 \%)$ de los pacientes venían derivados de otros $C A D$, con el objetivo de realizar la desintoxicación hospitalaria y una vez de alta seguir tratamiento ambulatorio en su CAD de referencia.

Una vez de alta hospitalaria los pacientes siguieron tratamiento ambulatorio en la UA-HCB o en su CAD de referencia. En los pacientes que se consideró necesario se utilizaron otros recursos psicoterapéuticos: Centros de Salud Mental (CSMA), Servicios Sociales (SS), Centros de Día, Organizaciones Deportivas, dispositivos de Larga Estancia residencial, etc.

El presente trabajo tiene el objetivo principal de conocer la evolución terapéutica y la mortalidad de los pacientes alcohólicos a los 14 años de su desintoxicación hospitalaria, después de haber seguido un tratamiento hospitalario de desintoxicación estándar.

Para el análisis de la mortalidad se han utilizado datos centralizados desde 1993 hasta finales del 2007, obtenidos del "Registre de Mortalitat de Catalunya de la Generalitat de Catalunya" ${ }^{15}$, que recoge todas las notificaciones de defunción al Registro Civil en la Comunidad Autónoma de Cataluña. Por la edad, gravedad orgánica y psiquiátrica y cronicidad de estos enfermos, los desplazamientos y cambios de residencia son excepcionales, por lo que hay una alta seguridad de disponer, mediante la consulta a este único registro, de datos fiables para toda la muestra.

\section{Análisis estadístico}

Frecuencias, medias y desviaciones típicas para la descripción de los sujetos. t de Student para la comparación de medias, análisis de la varianza en caso de más de dos medias y la Chi cuadrado $\left(\chi^{2}\right)$ para comparaciones entre variables cualitativas.
Análisis de regresión logística para identificar los factores relacionados con la mortalidad independientemente de la edad.

Comparación de la supervivencia de los pacientes entre distintos grupos según el estadístico de Mantel-Cox, incluyendo en la ecuación como covariables las variables que pudieran estar relacionadas con la muerte.

\section{RESULTADOS}

\section{Descripción de la muestra durante el ingreso}

La cohorte estudiada se compone de 52 hombres (57,1\%) y 39 mujeres $(42,9 \%)$, con una edad promedio de $41,2 \pm 11$ años (rango de 16 a 72 años) en el momento del ingreso en 1993, sin diferencias significativas entre ambos sexos.

La vía de ingreso es programada en $74,7 \%$ de casos, urgente en $11 \%$, judicial en $5,5 \%$ y derivados de otros dispensarios el $8,8 \%$ restante.

Los motivos de ingreso habían sido: tratamientos fracasados $(31,9 \%)$, politoxicomanía (11\%), falta de soporte socio-familiar $(9,9 \%)$, trastornos psiquiátricos o cognitivos $(7,7 \%)$ y patología orgánica importante $(8,8 \%)$. En $30,8 \%$ de casos existía más de un motivo para el ingreso.

La primera columna de la Tabla 1 muestra el elevado porcentaje de pacientes que presentaban criterios de comorbilidad psiquiátrica o neuropsicológica, de consumo de otras drogas además del alcohol (sin contar el tabaco), de antecedentes familiares de alcoholismo, de tratamientos alcohológicos y de ingresos previos (61,5\% en salas de hospitalización de tipo orgánico y 23,1\% de tipo psiquiátrico).

Sólo el 34,1\% están casados, estando solteros $(27,5 \%)$, separados $(29,7 \%)$ y viudos $(8,8 \%)$ el resto. están de baja, en paro, o son pensionistas el 7,7\%, $39,6 \%$ y $17,6 \%$ respectivamente.

Las primeras columnas de la Tabla 2 muestran la situación clínica de los pacientes a lo largo del ingreso y en el momento del alta hospitalaria. No existían diferencias significativas en el porcentaje de altas voluntarias según el centro de procedencia $(10,5 \%$ de los pacientes de otros CAD y $11,1 \%$ de los de la UA-HCB).

\section{Situación durante el seguimiento post-alta}

La Tabla 2 muestra la situación de los pacientes durante los 14 años posteriores al alta hospitalaria.

Durante este tiempo existen muchos cambios en el flujo de pacientes: abandonos de tratamiento, cambios de centro, derivaciones a otros dispositivos de las redes sanitaria o social, debido a las limitaciones de autonomía física y mental, y en algunos de los abandonos hay reinicios posteriores de tratamiento alcohológico, o bien se ponen en contacto con otros servicios hospitalarios por sus patologías orgánicas 
relacionadas con el consumo de alcohol. Finalmente se incrementa paulatinamente el número de los que fallecen.

Al final de la Tabla 1 se muestra el resultado del tratamiento a los 6 meses en los 72 pacientes que siguieron tratamiento en la UA-HCB.

\section{Mortalidad y factores asociados}

A los 14 años del alta hospitalaria habían fallecido 31 pacientes $(34,1 \%)$. El porcentaje de muertos entre hombres y mujeres $(32,7 \%$ versus $35,9 \%)$ o entre los tratados en la UA

Tabla 1. Diferencias entre pacientes vivos y fallecidos al final del seguimiento de 14 años.

\begin{tabular}{|c|c|c|c|c|c|}
\hline & & $\begin{array}{l}\text { Vivo } \\
(\mathrm{N}=60)\end{array}$ & $\begin{array}{l}\text { Fallecido } \\
(\mathrm{N}=31)\end{array}$ & \multicolumn{2}{|c|}{ Edad según la caracteristica inicia } \\
\hline CARACTERISTICAS INICIALES & $N=91$ & & & $\mathrm{Si}$ & No \\
\hline Edad en el momento de ingreso & $41,2 \pm 11$ & $39,1 \pm 10$ años & $45,4 \pm 11$ años ** & & \\
\hline Varones & $52(57 \%)$ & $58,3 \%$ & $54,8 \%$ & $39,9 \pm 11$ años & $43 \pm 10$ años \\
\hline Solteros & $25(27,5 \%)$ & $33,3 \%$ & $16,1 \%$ & $32,8 \pm 9$ & $44,4 \pm 9 * * *$ \\
\hline Estatus social bajo & $21(23 \%)$ & $18,3 \%$ & $32,3 \%$ & $47,6 \pm 12$ & $39,3 \pm 10^{* *}$ \\
\hline Nivel educativo bajo & $33(36 \%)$ & $33,3 \%$ & $41,9 \%$ & $44,2 \pm 12$ & $39,5 \pm 10 *$ \\
\hline Antecedentes familiares alcoholismo & $46(50 \%)$ & $46,7 \%$ & $60 \%$ & $41,8 \pm 11$ & $40,7 \pm 11$ \\
\hline Situación laboral Pensionista & $16(17 \%)$ & $11,7 \%$ & $29 \% *$ & $50,2 \pm 14$ & $39,3 \pm 9 * * *$ \\
\hline Motivación orgánica del tratamiento & $8(8,7 \%)$ & $63,3 \%$ & $67,7 \%$ & $47 \pm 14$ & $40,7 \pm 10$ \\
\hline Psicopatología & $37(40,6 \%)$ & $40 \%$ & $41,9 \%$ & $41 \pm 11$ & $41 \pm 11$ \\
\hline Abuso de otros tóxicos & $37(40,6 \%)$ & $43,3 \%$ & $35,5 \%$ & $38 \pm 9$ & $43,5 \pm 11 * *$ \\
\hline Ingresos previos & 77 (84\%) & $81,7 \%$ & $90,3 \%$ & $41,7 \pm 10$ & $38,9 \pm 13$ \\
\hline Tratamientos alcohológicos previos & $71(78 \%)$ & $78,3 \%$ & $77,4 \%$ & $40,5 \pm 10$ & $44 \pm 12$ \\
\hline Ingreso urgente & $10(10,9 \%)$ & $6,7 \%$ & $19,4 \%$ & $41,3 \pm 9$ & $41,2 \pm 11$ \\
\hline Alta voluntaria & $10(10,9 \%)$ & $11,7 \%$ & $9,7 \%$ & $35,3 \pm 11$ & $42 \pm 10$ \\
\hline Soporte familiar & $66(75 \%)$ & $76,7 \%$ & $64,5 \%$ & $39,6 \pm 10$ & $45,5 \pm 10 *$ \\
\hline Deterioro cognitivo (trastorno amnésico) & $40(43,9 \%)$ & $33,9 \%$ & $64,5 \%$ * & $46,1 \pm 10$ & $37,1 \pm 10^{* * *}$ \\
\hline Conciencia de dependencia & $28(30,7 \%)$ & $35 \%$ & $22,6 \%$ & $40,3 \pm 11$ & $41,7 \pm 10$ \\
\hline Sospecha de consumos encubiertos & $21(23 \%)$ & $23,3 \%$ & $22,6 \%$ & $39,8 \pm 12$ & $41,7 \pm 10$ \\
\hline Actitud colaboradora & $46(50 \%)$ & $55 \%$ & $41,9 \%$ & $41,5 \pm 10$ & $40,9 \pm 11$ \\
\hline Evolución positiva durante el ingreso & $46(50 \%)$ & $56,7 \%$ & $38,7 \%$ & $40,7 \pm 10$ & $41,7 \pm 11$ \\
\hline Utilización de otros dispositivos y recursos terapéuticos & $20(21,9 \%)$ & $18,3 \%$ & $29 \%$ & $40,6 \pm 10$ & $43,6 \pm 13$ \\
\hline Procedencia de otro CAD (no UA) & $19(20,8 \%)$ & $18,3 \%$ & $25,8 \%$ & $44,1 \pm 9$ & $40,5 \pm 11$ \\
\hline Indicación de alcoholúrias & $64(70 \%)$ & $71,7 \%$ & $67,7 \%$ & $41,2 \pm 11$ & $41,2 \pm 11$ \\
\hline Indicación de terapia grupal & $10(10,9 \%)$ & $13,3 \%$ & $6,5 \%$ & $45,8 \pm 8$ & $40,7 \pm 11$ \\
\hline Indicación de disulfiran & $80(87,9 \%)$ & $90 \%$ & $83,9 \%$ & $40,9 \pm 10$ & $43,9 \pm 16$ \\
\hline Indicación de antidepresivos & $15(16,4 \%)$ & $10 \%$ & $29 \% *$ & $40,7 \pm 12$ & $41,3 \pm 10$ \\
\hline Situación en el seguimiento entre los 72 pacientes UA (no derivados) & $N=72$ & $N=49$ & $\mathrm{~N}=23$ & & \\
\hline Recaidas a los 6 meses (considerando los abandonos como recaídas). & 60 & $77,6 \%$ & $95,7 \%$ * & $40,4 \pm 11$ años & $40,9 \pm 9$ años \\
\hline Alta terapéutica a los 2 años & 4 & $8,2 \%$ & $0 \%$ & & \\
\hline Resultado positivo (alta o en tratamiento) del tratamiento a los 2 años & 26 & $36,7 \%$ & $34,8 \%$ & & \\
\hline
\end{tabular}

Pruebas estadisticas: $t$ de Student para la comparación de medias y $\mathrm{Chi}^{2}$ para la de frecuencias.

${ }^{*} p<0,05^{* *} p<0,01{ }^{* *} p<0,001$.

CAD: Centro de Atención a las Drogodependencias; UA: Unidad de Alcohologia; Otros dispositivos terapéuticos: Centro de Salud Mental (CSMA), Servicios Sociales, Centro de Dia, organizaciones deportivas, dispositivos de Larga Estancia (Residencias, Comunidades Terapeuticas). 
Tabla 2. Situación clínica de los pacientes a lo largo del periodo de seguimiento.

\begin{tabular}{|c|c|c|c|c|c|c|c|c|}
\hline & $\begin{array}{l}\text { Dia de } \\
\text { ingreso }\end{array}$ & Día de alta & Mes 1 & Mes 6 & Mes 12 & Año 2 & Año 3 & Año 14 \\
\hline Tratamiento en otros CAD & 19 & $\begin{array}{l}17 \text { (3 además con otros } \\
\text { recursos) }\end{array}$ & 17 & 17 & 17 & 14 & 14 & 10 \\
\hline Abandono del tratamiento & 0 & $\begin{array}{c}10 \text { (2 de otros } C A D, 8 \text { de } \\
\text { la UA) }\end{array}$ & 18 & 31 & 37 & 45 & 36 & 30 \\
\hline $\begin{array}{l}\text { Derivado a otros } \\
\text { dispositivos }\end{array}$ & 0 & 0 & 0 & 0 & 0 & 0 & 2 & 2 \\
\hline Tratamiento en la UA-HCB & 72 & $\begin{array}{l}64 \text { (17 además con otros } \\
\text { recursos) }\end{array}$ & 56 & 43 & 36 & 17 & 19 & $\begin{array}{c}6 \text { (4 pacientes crónicos y } 2 \text { desaparecidos } \\
\text { que vuelven a la UA) }\end{array}$ \\
\hline $\begin{array}{l}\text { Tratamiento en otros } \\
\text { servicios del HCB }\end{array}$ & 0 & 0 & 0 & 0 & 0 & 0 & 0 & $\begin{array}{c}5 \text { (abandonos que vuelven al HCB, no } \\
\text { a la UA }\end{array}$ \\
\hline Alta del tratamiento & 0 & 0 & 0 & 0 & 0 & 9 & 9 & 7 \\
\hline Total & 91 & 91 & 91 & 91 & 91 & 91 & 91 & 91 \\
\hline
\end{tabular}

CAD: Centro de Atención a las Drogodependencias. UA-HCB: Unidad de Alcohologia del Hospital Clinico de Barcelona. CSMA: Centro de Salud Mental; Día de alta: Finalización programada de la desintoxicación (aproximadamente a los 21 dias del ingreso). Otros recursos: utilización de otros recursos terapéuticos en 20 pacientes de forma complementaria al seguimiento de su tratamiento alcohológico (5 pacientes con CSMA, 9 con Servicios Sociales y 2 también con CSMA, uno con Centros de Dia, 2 con Organizaciones Deportivas, y un paciente a un dispositivo de Larga Estancia residencial).

o por otras CAD (31,9\% versus $42,1 \%)$ no presenta diferencias estadísticamente significativas.

La edad en el momento de la muerte era de $51,4 \pm 12$ años (rango de 25 a 75 años). Los pacientes fallecidos durante los 14 años de seguimiento eran significativamente mayores al inicio del estudio (ingresados en 1994) que los que sobrevivieron $(45,4 \pm 11$ años versus $39,1 \pm 10$ años; $t=2,7 ; p=0,008)$.

En general existe un incremento paulatino de la mortalidad a medida que aumenta la edad en el momento del ingreso: $19 \%$ entre los pacientes situados en el primer cuartil de edad, $28 \%$ en el segundo cuartil, $39,1 \%$ en el tercero y $50 \%$ de los situados en el cuarto cuartil.

La Tabla 1 muestra las diferencias, tanto al inicio como al final del periodo de seguimiento, entre los pacientes que fallecieron y los que siguieron vivos durante ese periodo.

Los pacientes fallecidos se caracterizaban al inicio del tratamiento por una mayor frecuencia de deterioro cognitivo y del porcentaje de pensionistas, rasgos que están ambos asociados a una mayor edad, tal y como se comprueba en las dos últimas columnas de la Tabla 1. También presentaban una peor evolución en el tratamiento.

Los pacientes de mayor edad ya no son solteros y han consumo otros tóxicos con menor frecuencia, aspectos que no tienen una relación aparente con una mayor mortalidad.

Pero estos pacientes mayores también tienen menos soporte familiar, un nivel social y educativo mas bajo, más antecedentes de alcoholismo, una actitud menos colaboradora, una evolución menos positiva y una menor conciencia de dependencia durante el ingreso de desintoxicación, que acostumbra a ser urgente más a menudo. Aunque estas diferencias no llegan a la significación estadística sí configuran un patrón psicosocial coherente con un mayor riesgo de muerte.
Finalmente se observa que los pacientes que fallecieron presentaban durante los dos años de tratamiento ambulatorio un mayor porcentaje de recaídas alcohólicas o abandonos del tratamiento antes de los 6 meses y que no lograron nunca el alta terapéutica.

\section{Análisis multivariado}

Se ha realizado un análisis de regresión logística binaria por pasos hacia delante para intentar predecir los factores que influyen en la muerte antes de finalizar los 14 años de seguimiento, separando el efecto de la edad.

Se han incluido las variables que han mostrado tener individualmente relación con la mortalidad (deterioro neuropsicológico, estatus de pensionista, prescripción de antidepresivos y recaída/abandono antes de los 6 meses) y las que tenían indicios clínicos de ser de mal pronóstico terapéutico (antecedentes familiares de alcoholismo, ingresos previos, baja conciencia de dependencia, baja colaboración y mala evolución durante el ingreso, y nivel educativo y estatus social bajos), además de la edad al inicio del estudio.

El resultado muestra que en el modelo que clasifica correctamente a un mayor porcentaje de los pacientes que fallecen, tan sólo las tres primeras variables (deterioro neuropsicológico, estatus de pensionista, prescripción de antidepresivos) tienen significación estadística en la ecuación de regresión, sin que la edad intervenga por si misma.

Parece por tanto que los pacientes con deterioro neuropsicológico, estatus de pensionista y que reciben antidepresivos sufren una especie de envejecimiento prematuro, probablemente provocado por el alcohol y que ello redunda en una mayor probabilidad de muerte temprana. 
También se ha realizado un análisis cualitativo, mediante la prueba de Chi cuadrado, de estas tres variables con la mortalidad, estratificado por la edad al inicio del estudio, dicotomizada en mayor o menor de 41 años.

El resultado es que en los pacientes más jóvenes (menores de 41 años) la presencia de deterioro cognitivo, pero no de las otras dos variables, tiene relación con un mayor riesgo de muerte (presencia de deterioro cognitivo en el $20 \%$ de los pacientes vivos frente al 54,5\% en los que fallecen; $\mathrm{Chi}^{2}=$ $4,9 ; p=0,026)$.

En cambio en los pacientes mayores el deterioro cognitivo no se relaciona con un mayor riesgo de muerte, relación que sí sucede en caso de ser pensionista (12\% de los pacientes vivos y $45 \%$ de los que fallecen; $\mathrm{Chi}^{2}=6,1 ; p=0,013$ ), o recibir antidepresivos ( $8 \%$ de pacientes vivos y $30 \%$ de los que fallecen; $\left.\mathrm{Chi}^{2}=3,6 ; \mathrm{p}=0,05\right)$.

El análisis cualitativo estratificado por edad de la mortalidad según las recaídas y abandonos durante los 6 primeros meses de los 72 pacientes en tratamiento en la UA-HCB revela que sólo en los pacientes mayores de 41 años se produce un incremento de la mortalidad en el caso de haber recaído en el consumo de alcohol (de 0\% de los 6 pacientes que no recaen al 55,6\% de los 27 que sí recaen; $\mathrm{Chi}^{2}=6,1$; $p=0,01)$. En los más jóvenes no existen diferencias significativas (16,7\% versus $21,2 \% ; \mathrm{Chi}^{2}=0,06 ; \mathrm{p}=$ n.s.)

En resumen, la presencia de deterioro cognitivo $(O R=2,9$; $p=0,02)$ conjuntamente con una edad superior a 41 años $(O R=1,9 ; p=n$.s. $)$ son factores de riesgo de muerte $\left(R^{2}\right.$ de $\operatorname{Cox}=0,1$; porcentaje correctamente clasificado $=66,7 \%$ ). También en los pacientes tratados en la UA-HCB las recaídas son un elemento de mal pronóstico vital en los mayores. Pero en el caso de los pacientes más jóvenes (menos de 41 años) la Odds Ratio (OR) de muerte que tienen los pacientes deteriorados cognitivamente frente a los conservados $(O R=4,8$; $p=0,03$ ) duplica a la de los pacientes mayores.

La edad también confunde la relación del estatus de pensionista con la mortalidad: en los pacientes jóvenes no existe relación (sólo 4 pensionistas, el 100\% vivos a los 14 años, $\mathrm{Chi}^{2}=1,3 ; \mathrm{p}=$ n.s.), mientras que en los mayores (de 41 años) sí la hay: el 75\% de pensionistas ha muerto, por tan sólo el $33,3 \%$ de los no pensionistas $\left(\mathrm{Chi}^{2}=6,1 ; p=0,01\right)$.

En cambio la edad no influye en el riesgo de muerte según se prescriban o no antidepresivos: la odds ratio global de morir de los pacientes a los que se indicó antidepresivos frente a los que no se les indicó es de 3,6 (Chi $=5,3 ; p=$ $0,02)$, pero esta $O R$ es más acusada en los pacientes mayores $\left(O R=4,9, C h i^{2}=3,6 ; p=0,05\right)$ que en los jóvenes $(O R=2,9$; $\mathrm{Chi}^{2}=1,6 ; p=$ n.s.).

\section{Análisis de supervivencia}

Por último se realiza un análisis de supervivencia de los días hasta el fallecimiento introduciendo la edad como covariable en un modelo de regresión de Cox y ajustándola respecto al deterioro cognitivo, los tratamientos alcohológicos e ingresos previos, la existencia de psicopatología, la consciencia de dependencia alcohólica, el estatus social, la evolución durante el ingreso de desintoxicación, la indicación de antidepresivos y la existencia de recaídas antes de los 6 meses de seguimiento. Mediante el método de introducción hacia delante por pasos, el modelo que explica mejor y de forma más significativa los días de supervivencia es el que contiene las variables evolución positiva durante el ingreso (Wald $=4,5 ; p=0,03 ; 0 R=2,2)$, prescripción de antidepresivos (Wald $=5,9 ; p=0,01 ; O R=0,3)$ y la edad (Wald = 7,6; $p=0,006 ; O R=1,04)$, que influyen significativamente en la supervivencia.

La gráfica 1 muestra la diferencia en las curvas de supervivencia entre pacientes con o sin tratamiento antidepresivo usando el resto de variables relacionadas como covariantes.

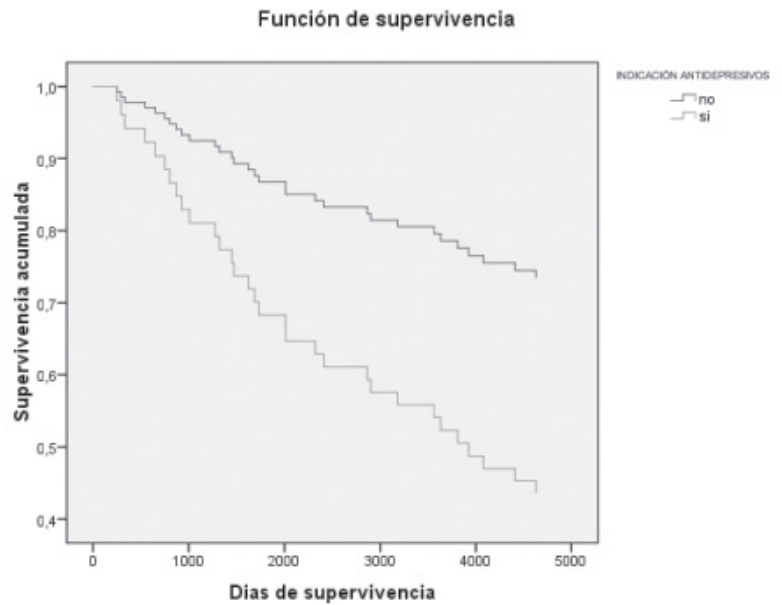

Gráfica 1. Curvas de supervivencia entre pacientes con o sin prescripción de antidepresivos usando el resto de variables relacionadas como covariantes.

\section{DISCUSIÓN}

\section{Características de los pacientes, grado evolutivo de su dependencia y mortalidad}

Se confirma que los pacientes alcohólicos que precisan de un ingreso de desintoxicación en una sala de Psiquiatría son relativamente jóvenes, de edad similar a los que acuden voluntariamente a realizar tratamientos ambulatorios. Por otra parte son mucho más jóvenes (41 versus 50 años) que los que son detectados a raíz de su ingreso en hospitales generales por enfermedades orgánicas relacionadas con su consumo de alcohol ${ }^{13}$. Son por tanto pacientes con una dependencia alcohólica de relativamente pocos años de evolución.

Sin embargo tienen una elevada proporción de trastornos psiquiátricos y neuropsicológicos comórbidos, complicaciones psico-sociales y familiares y pérdida de la capacidad para trabajar o del soporte del entorno sociofamiliar, aunque el daño orgánico es poco significativo en el momento del ingreso. 
Aunque sus alteraciones físicas son leves, más parecidas a las de los alcohólicos en régimen ambulatorio que a las de los hospitalizados orgánicamente, tienen más gravedad en su dependencia alcohólica, por la comorbilidad psiquiátrica.

Su seguimiento ambulatorio es malo. Solo el 10\% de pacientes logra el alta terapéutica a los 2 años. A pesar de la elevada utilización de otros recursos Sociales y de Salud Mental estos enfermos tienen una baja adherencia a los tratamientos. Probablemente existe también un elevado porcentaje de recaídas en el consumo de alcohol.

Su mortalidad a los 14 años es del 34,1\% a una edad promedio de 51,4 años. Esta mortalidad se sitúa de forma intermedia entre la de los pacientes ambulatorios y la de los ingresados por causas orgánicas (32\% a los 20 años y $30 \%$ a los 4 años respectivamente). Sin embargo la edad en el momento de morir es más joven que la de los alcohólicos ingresados por causas orgánicas (57,5 años) ${ }^{12}$.

Una posible explicación es que el deterioro neuropsicológico es más frecuente en los pacientes estudiados (43,9\% frente al $15,7 \%$ de los pacientes ingresados por causa orgánica). Otra explicación complementaria es que muchos alcohólicos, en los que predominan los trastornos conductuales, sufren muertes precoces (alrededor de los 50 años, precisamente la de nuestra muestra), debidas a problemas neuropsicológicos (accidentes, suicidios, traumatismos, demencias 0 síndromes amnésicos alcohólicos, complicaciones neuropáticas, etc.). En cambio los alcohólicos que se detectan cuando ingresan por causas orgánicas son supervivientes de una cohorte que ya ha sufrido una importante mortalidad previa.

Ello provoca un sesgo: minimiza la gravedad de la dependencia alcohólica de los que siguen vivos, que parecen bebedores sociales de toda la vida, a la vez que acrecienta la gravedad relativa de las consecuencias de salud de los alcoholicos hospitalizados por causas físicas, pues en realidad mueren antes los pacientes menos "orgánicos" y más "psiquiátricos".

Precisamente las enfermedades del sistema nervioso habian sido una de las principales causas de muerte de los alcohólicos detectados por causa orgánica ${ }^{12}$ y en la presente muestra el deterioro cognitivo es el factor que por si sólo mejor predice la mortalidad a los 14 años.

Un mejor cumplimiento del tratamiento a medio plazo (6 meses) se acompaña de menores porcentajes de mortalidad y obtener el alta terapéutica (4 pacientes) se asocia siempre con supervivencia. Pero también es muy probable que los pacientes que ya sufren algún deterioro neuropsicológico previo tengan menor capacidad para mantenerse abstinentes y en tratamiento, precisamente debido a que su deterioro interfiere con el control de la conducta. Ello sugiere que los tratamientos habituales del alcoholismo una vez superada una barrera en que se han producido ciertos daños irreversibles, especialmente neuropsicológicos, además de ser infructuosos no pueden retrasar la muerte precoz del paciente. En el grupo de pacientes estudiados y que falleció, esta barrera estaría en torno a los 45 años y las muertes se producirian alrededor de los 50 años, probablemente por trastornos neuropsicológicos.
Superada esta barrera existiría otro grupo de alcohólicos, los más "sociales", sin tantos trastornos neuropsicológicos, pero con enfermedades orgánicas (hepáticas y neoplásicas), que serían los que menos acudirían a solicitar ayuda y que se detectarían de forma tardía, por sus complicaciones orgánicas y con una escasa esperanza de vida, a los pocos años del ingreso, que se situaría en torno a los 57 años ${ }^{12}$.

\section{Factores que influyen en la mortalidad}

La presencia de deterioro cognitivo y de psicopatología son rasgos definitorios de estos enfermos, pero de los dos sólo el deterioro se muestra relacionado con el riesgo de muerte durante los años siguientes al ingreso para desintoxicación. Indudablemente la edad tiene también un papel central y ambos factores, edad y deterioro cognitivo, están interrelacionados.

Sin embargo nuestros datos sugieren que hay una serie de indicadores indirectos de mal funcionamiento neuropsicológico, sobretodo entre los pacientes más jóvenes y que serían coherentes con la antigua hipótesis del envejecimiento prematuro en los pacientes alcohólicos con deterioro cognitivo ${ }^{16-18}$. En otros estudios también se extrae esta conclusión ${ }^{7,12}$.

Entre estos indicadores hay: i) la falta de una evolución positiva del paciente durante el ingreso de desintoxicación, ii) la presencia de síntomas de deterioro cognitivo, iii) el ser pensionista y iv) recibir antidepresivos. Los dos primeros factores influyen sobretodo en los pacientes más jóvenes, mientras que los últimos afectan más al riesgo de muerte de los mayores.

Globalmente la impresión es que todos estos factores se relacionan de forma indirecta con la existencia de un deterioro orgánico: La prescripción de antidepresivos podría indicar la presencia de síntomas que encubririan un deterioro cognitivo latente e incluso que estos síntomas depresivos favorecieran otros problemas orgánicos ${ }^{19}$, el ser pensionista indicaría un menor grado de autonomía física y cognitiva y la mala evolución durante el ingreso sería el resultado de una menor capacidad mental para entender su enfermedad y modificar sus hábitos.

El hecho de que la mejor evolución terapéutica (menos recaídas o abandonos del tratamiento, más altas terapéuticas y seguramente mayor mantenimiento de la abstinencia) se asocie con mantenerse vivo, aunque no sea un factor que aparezca en el análisis multivariado, probablemente indica que esta mejor evolución terapéutica se asocia precisamente con la menor presencia de deterioro psico-orgánico.

\section{Causas de la muerte}

No disponemos de información directa acerca de las causas de muerte, pero posiblemente se deben con más frecuencia a los trastornos psiquiátricos (suicidio, accidente, traumatismo, malas condiciones de vida, etc.) que provocan conductas de riesgo y un menor autocontrol, que a los 
aspectos puramente orgánicos. De los 11 pacientes fallecidos y que eran menores de 41 años al inicio del estudio no sabemos la causa de la muerte en 5 . Pero en el resto, pocos meses antes de la muerte, revisando los archivos de nuestro hospital hallamos problemas pancreáticos en un paciente, hepáticos en otro y en otros 3 había diversas urgencias, precisamente psiquiátricas. En el paciente restante constaba claramente la causa de muerte por suicidio (traumático).

En conclusión, los pacientes alcohólicos de peor evolución, con más antecedentes de tratamientos fracasados, menor conciencia de su enfermedad y menor capacidad para modificar su conducta, utilizan abundantes recursos psiquiátricos intensivos y dispositivos sociales. Aunque son comparativamente más jóvenes y menos afectados orgánicamente que los alcohólicos detectados en los hospitales generales, tienen un perfil más neuro y psicopatológico y presentan también importantes tasas de mortalidad a edades tempranas.

Para los clínicos seguramente no es ninguna sorpresa, pero los datos presentes apoyan la idea de que el seguimiento terapéutico de estos pacientes debe tener en cuenta este riesgo vital además de la patología psiquiátrica y socio-familiar de base.

Una estrategia para ello ha de ser la coordinación de los servicios sanitarios, sociales, y a veces judiciales, implicados, y que éstos tengan conocimientos sobre los riesgos de los pacientes alcohólicos que presentan rasgos psicopatológicos y deterioro cognitivo incipiente.

Dentro de los sanitarios, tanto los médicos de atención primaria, como los especialistas o los servicios de urgencias, han de poder tener información instantánea, con la ayuda de la informática, de que éstos pacientes, aunque relativamente jóvenes y aparentemente con pocas alteraciones físicas, son de alto riesgo vital. En el momento en que cualquiera de ellos detecte a estos enfermos (muchas veces son los servicios de urgencias) deberían contar con protocolos para la contención y derivación rápida al servicio de drogodependencias y/o salud mental correspondiente.

Una vez retornados a estos servicios se debe potenciar la reducción de daños y el cuidado orgánico, pero también el control de las conductas impulsivas desadaptativas, probablemente haciendo seguimientos más intensos y coordinados, otra vez, con el resto de agentes de salud.

Finalmente quizás haya que recordar que las complicaciones orgánicas en si mismas no son el criterio de gravedad del alcoholismo, pero que el deterioro psico-orgánico y psico-social también mata, y a edades tempranas ${ }^{20}$, incluso a sus familiares ${ }^{21}$. Y el alcohol por si sólo, sin dependencia ni trastornos psiquiátricos, también ${ }^{20}$.

\section{Agradecimientos}

Al Registre de Mortalitat de Catalunya del Servei d'Informació i Estudis (Direcció General de Recursos Sanitaris, Departament de Sanitat i Seguretat Social, Generalitat de
Cataluña), gracias a cuya colaboración ha sido posible identificar a los pacientes que habian fallecido y saber la fecha de su defunción.

\section{REFERENCIAS}

1. Revuelta E, Godoy P, Farreny M. Evolución de la mortalidad atribuible al consumo de alcohol en Cataluña, 1988-1997. Aten Primaria 2002; 30: 112-118.

2. Redondo Calderón JL, Luna del Castillo JD, Jiménez Moleón JJ, Lardelli Claret P, Gálvez Vargas R. Evolución de la mortalidad por accidentes de tráfico en España, 1962-1994. Gac Sanit 2000; 14: 7-15.

3. Sjögren $H$, Eriksson A, Bröstrom G, Alm K. Quantification of alcohol-related mortality in Sweden. Alcohol 2000; 35: 601-11.

4. Shultz JM, Rice DP, Parker DL. Alcohol-related mortality and years of potential life lost United States, 1987. MMWR 1990; 39: 173-8.

5. Rossow I, Amundsen A. Alcohol abuse and mortality: a 40-year prospective study of Norwegian conscripts. Soc Sci Med 1997; 44: 261-7.

6. Andreasson S, Brandt L. Mortality and morbidity related to alcohol. Alcohol \& Alcoholism 1997; 32: 173-178.

7. Gual A, Lligoña A, Costa S, Segura L, Colom J. Tratamiento del alcoholismo y su impacto a largo plazo. Resultados a 10 años de un estudio longitudinal prospectivo de 850 pacientes. Med Clin 2004; 123: 364-9.

8. Fichter MM, Quadflieg N, Fischer UC. Severity of alcohol-related problems and mortality: results from a 20 -year prospective epidemiological community study. Eur Arch Psychiatry Clin Neurosci 2011 Jun; 261:293-302.

9. Gual A, Bravo F, Lligoña A, Colom J. Treatment for Alcohol Dependence in Catalonia: Health Outcomes and Stability of Drinking Patterns over 20 Years in 850 Patients. Alcohol Alcohol 2009 44:409-15.

10. Monras M, Ortega L, Mondon S, Gual A. Detección y tratamiento de las drogodependencias en un Hospital General. Adicciones 2003; 15: 331-340.

11. Monras M, Ortega L, Mondon S, Balcells M, Gual A. Enfermedades relacionadas con el consumo de alcohol: seguimiento a los dos años de la hospitalización. Medicina Clínica 2004; 123: 521-6.

12. Monras M, Mondon S, Ortega L, Gual A. Alcoholismo en el hospital general: Mortalidad y hospitalizaciones a los 4 años de su detección. Med Clin 2005; 125:441-7.

13. Monras M, Garcia S, Torres M, Gual A, Ortega L. El ingreso hospitalario en el tratamiento de los alcohólicos cronificados. Adicciones 1998; 10: 151-160.

14. Chwastiak LA, Rosenheck RA, Desai R, Kazis LE. Association of psychiatric illness and all-cause mortality in the National Department of Veterans Affairs Health Care System. Psychosom Med 2010; 72: 817-22.

15. Registre de Mortalitat de Catalunya, Servei d'Informació i Estudis, Direcció General de Recursos Sanitaris, Departament de 
Sanitat i Seguretat Social. Generalitat de Catalunya. Barcelona, 1993-2007.

16. Uekermann J, Daum I. The neuropsychology of alcoholism. Advances in psychology research Vol 49 [e-book]. Hauppauge, NY US: Nova Science Publishers; 2007:167-187.

17. Ryan, C., Butters, N. Learning and memory impairments in young and old alcoholics: Evidence for the premature-aging hypothesis. Alcoholism: Clinical and Experimental Research 1980; 4: 288-293.

18. Kramer JH, Blusewicz MJ, Preston KA. The premature aging hypothesis: old before its time? J Consult Clin Psychol 1989; 57: 257-62.

19. Taylor V., McKinnon MC, Macdonald K, Jaswal G, MacQueen GM. Adults with mood disorders have an increased risk profile for cardiovascular disease within the first 2 years of treatment. Can. J. Psychiatry 2010; 55: 362-368.

20. Anderson, P. i Baumberg, B. El alcohol en Europa. Institute of Alcohol Studies 2006 Londres.

21. Webb RT, Abel KM, Pickles AR, Appleby L, King-Hele SA, Mortensen PB. Mortality risk among offspring of psychiatric inpatients: a population-based follow-up to early adulthood. Am J Psychiatry 2006; 163: 2170-7. 
